DOI: $10.4274 /$ jarem.galenos.2021.3926

J Acad Res Med 2021;11(2):137-42

\title{
Functional and Radiological Outcomes of Intra- articular Calcaneus Fractures: A Comparison Between Accompanying Skeletal Injuries and Isolated Fractures
}

\author{
(1) Esra Çirci, (1) Alican Barış
}

University of Health Sciences Turkey, İstanbul Training and Research Hospital, Clinic of Orthopedics and Traumatology, İstanbul, Turkey

Cite this article as: Çirci E, Barış A. Functional and Radiological Outcomes of Intra-articular Calcaneus Fractures: A Comparison Between Accompanying Skeletal Injuries and Isolated Fractures. J Acad Res Med 2021;11(2):137-42

\begin{abstract}
Objective: This study aimed to compare between accompanying skeletal injuries and isolated fractures in terms of the functional and radiological results of treating intra-articular displaced calcaneus fractures with calcaneal locking compression plate.

Methods: Thirty-three patients with displaced intra-articular fracture of the calcaneus were included in the study. All patients were operated on using the lateral extensile approach with open reduction and internal fixation technique with a follow-up of more than four years. Böhler angle and crucial angle of Gissane were documented on preoperative and post-operative radiographs. The American Orthopaedic Foot and Ankle Society (AOFAS) hindfoot scores were evaluated.

Results: There was no significant difference between patients with and without accompanying injuries in terms of the duration of follow-up ( $p=0.646)$, complication rate $(p=0.797)$ and final outcome distribution ( $p=0.309)$. In patients with no additional injuries, AOFAS scores $(p=0.036)$ and postoperative Böhler angle $(p=0.007)$ were significantly higher. The post-operative Gissane angle did not significantly differ between the two groups $(p=0.241)$.

Conclusion: In our study, we concluded that the accompanying injuries in the calcaneus will adversely affect the radiological results and functional outcomes. During the management of calcaneus fractures, the accompanying injuries and their negative effects on the healing process should be taken into consideration.
\end{abstract}

Keywords: Calcaneus fracture, bilaterally, accompanied injury, radiological evaluation, functional evaluation

\section{INTRODUCTION}

The calcaneus is the most commonly fractured tarsal bone, representing $60 \%$ of all tarsal fractures and nearly $2 \%$ of all fractures (1). The subtalar joint is often involved at a rate of approximately $75 \%$. In the young adult population, most intraarticular calcaneus fractures caused by axial loading result from traffic accidents or falls from a height and often have associated damages. An axial load power affects the posterior facet of the calcaneus through the talus, and shear powers are focused through the posterior facet toward the medial wall of the calcaneus (2).

The computerised tomography scans describe comminution and displacement of the posterior facet. Sanders classification is used to assess intra-articular calcaneus fractures, owing to its precision regarding the location and number of fracture lines through the posterior facet (3).

ORCID IDs of the authors: E.Ç. 0000-0001-6515-4324; A.B. 0000-0001-6031-6777. 
Calcaneal fractures are a considerable challenge for orthopedists because the displays of the fracture are various, and complications commonly occur. Anatomical reduction with stable fixation is the treatment of choice for displaced intra-articular fractures (4). The lateral approach is commonly used for open reduction and internal fixation (ORIF) of complex calcaneus fractures. This exposure provides anatomical reduction of the subtalar joint, restoration of the calcaneal morphology and stable locking compression plate fixation; therefore, early range of motion is encouraged. Anatomical reduction and stable fixation to avoid complications improve the functional outcomes, decrease morbidity and shorten recovery time (5).

Calcaneus fractures are severe high-energy injuries with the potential for considerable morbidity. Accompanying injuries and bilateral calcaneal fractures should be considered in the diagnosis and treatment process. The socioeconomic burden of calcaneus fractures is significant.

This study aimed to compare between accompanying skeletal injuries and isolated fractures in terms of the functional and radiological results of the treatment of intra-articular displaced calcaneus fractures with calcaneal locking compression plate. Our hypothesis was that ORIF of displaced intra-articular calcaneal fractures in patients without additional injuries would result in better outcomes.

\section{METHODS}

This study was approved by the Clinical Research Ethics Committee of Istanbul Training and Research Hospital (decision no: 886 , date: 25.11 .2016 ), and informed consent was obtained from the patients.

All patients admitted from January 2011 to November 2015 with a diagnosis of unilateral or bilateral displaced intra-articular fractures of the calcaneus were considered for inclusion. A consecutive series of 33 patients with displaced intra-articular fractures were treated by open reduction through an extended lateral approach and internal fixation with a locking compression plate and followed for more than four years post-operatively.

The study included three (9.1\%) females and $30(90.9 \%)$ males. The average age of the patients at the time of surgery was $38.5 \pm 10.6$ years (range: 20-56). Both calcanei were injured in eight male patients (three of them had accompanying injuries, and five of them had isolated bilateral calcaneus fractures).

The exclusion criteria were patients who were treated conservatively and the lack of surgical conditions. Patients with no intra-articular calcaneus fractures, those with open fractures, those who had a previous foot or ankle surgery, accompanying neural or vascular pathology, neuropathic foot, and pathologic fractures of the calcaneus, those younger than 18 years and those unable to come for follow-ups were excluded from this study.

For the precise assessment of intra-articular calcaneus fractures, preoperative conventional radiographs and computerised tomography with three-dimensional analysis were used. Sanders classification is used to assess intraarticular calcaneus fractures, owing to its precision regarding the location and number of fracture lines through the posterior facet. Fractures were categorised according to Sanders classification as follows. Type I fractures include all minimally displaced $(\leq 2 \mathrm{~mm}$ ) articular fractures, regardless of the number of fracture lines and fragments present. Type II fractures are displaced two-part fractures of the posterior facet with one primary fracture line that can be accompanied by one or more accessory fracture lines that do not involve the posterior articular facet. Type III fractures include threepart fractures of the posterior facet from two primary fracture lines commonly accompanied by a central area of depression. Type IV fractures involve three or more primary fracture lines, resulting in four or more articular fragments with severe comminution (3).

Surgery was carried out after the swelling diminished and skin wrinkles emerged. The average duration between the injury and surgery was five days (range: 2-11 days). Antibiotic prophylaxis was administered. The operations were performed under spinal anaesthesia with the patient in the lateral decubitus position, and a tourniquet was applied.

The surgery was performed using the lateral extensile approach (Figure 1). The full-thickness skin flap was retracted using one $\mathrm{K}$-wire in the fibula and two in the talus. Anatomical reduction was achieved, and the Böhler and Gissane angles were restored. A calcaneal plate (TST ${ }^{\oplus}$ locking calcaneus plate) was used (Figure 2). To maintain the height of the posterior facet, calcium phosphate cement $\left(\right.$ NeoCement $\left.{ }^{\circledR}\right)$ and allograft cancellous chips (Gencure ${ }^{\circledR}$ ) were supplemented according to the surgeon's preference in the presence of bone defects. The skin was closed using Ethilon 3.0. A wound drainage was used. A pressure bandage was applied for a few days.

Sutures were removed three weeks after the operation. Motion exercises were avoided during that time to lessen the shear forces under the flap. Protection was supplied using a removable posterior splint. Then, active range of motion of the ankle and subtalar joint was started. No weight-bearing was approved for the initial six to eight weeks, and then, gradual weight-bearing was introduced. Full weight-bearing was allowed after 12 weeks.

Patients were divided into two groups according to the presence of accompanying skeletal injuries.

Patients were asked to complete a questionnaire to calculate the outcome score, the disease-specific American Orthopaedic Foot and Ankle Society (AOFAS) hindfoot score for patient satisfaction, at the last follow-up (6). Based on the AOFAS hindfoot score, a total score ranging from 90 to 100 points was considered 'excellent', a range from 75 to 89 points was considered 'good', a range from 50 to 74 points was considered 'fair' and a score below 49 points was considered 'poor'.

Böhler angle and crucial angle of Gissane were documented on preoperative and post-operative radiographs at the last follow- 
up. The comparison views were used for surgical guidance in restoring normal anatomy in the contralateral foot in the unilateral cases.

\section{Statistical Analysis}

For the descriptive statistics of the data, mean, standard deviation, frequency, median lowest and highest values and ratio values were used. The distribution of the variables was measured using the Kolmogorov-Smirnov test. The Mann-Whitney $U$ test was used for the analysis of quantitative independent data. The Wilcoxon test was used for the analysis of the quantitativedependent data. The chi-square test was used for the analysis of the qualitative independent data. Fisher's Exact test was used when the chi-square test conditions were not provided. The data were analysed using the Statistical Package for Social Sciences (SPSS) version 22.0 (SPSS Inc. Chicago, IL). A p value less than 0.05 was considered statistically significant.

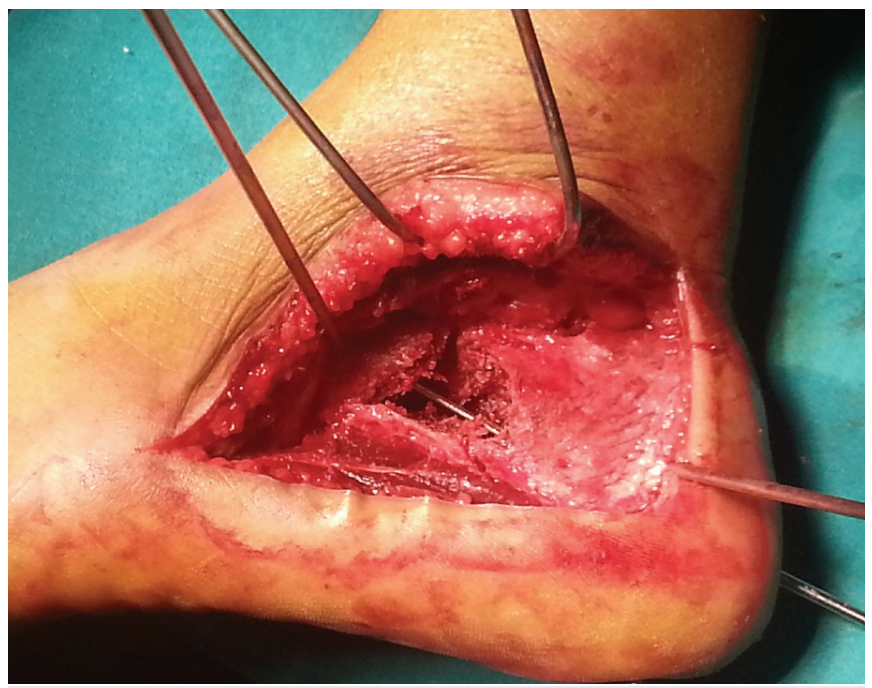

Figure 1. Lateral extensile approach

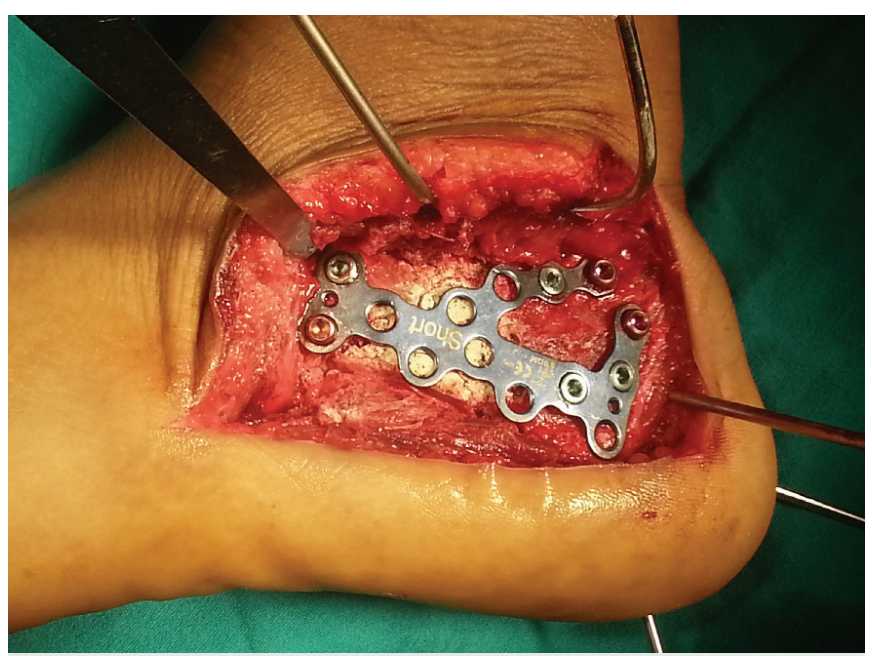

Figure 2. Placement of the low-profile locking anatomic plates with grafting

\section{RESULTS}

The mechanism of trauma is summarised in Table 1. The mean follow-up after surgery was 66.7 \pm 16.7 months (range: 48-96 months). According to Sanders classification, 17 fractures (41.5\%) were Sanders II, 14 fractures (34.1\%) were Sanders III and 10 fractures (24.4\%) were Sanders IV.

Accompanying injuries were present in 14 patients (42.4\%): Four patients had ipsilateral lower leg fracture; one patient, vertebra fracture (the patient with bilateral calcaneus fracture); three patients, vertebra and ipsilateral lower leg fracture (two of these patients with bilateral calcaneus fracture); one patient, upper extremity and lower leg fracture; five patients, isolated bilateral calcaneus fractures (without other injuries).

A total of 11 patients (33.3\%) had complications in this study. Six patients (18.2\%) had Sudeck's atrophy. Four patients (12.1\%) had superficial wound infection and delayed healing. One patient (3.0\%) had screw cut-out. The screw was removed, and acceptable functional improvement was achieved. The patient's complaints regressed.

Sudeck's atrophy developed six to fifteen weeks after the operation in six patients. Physical therapy exercises were increased. Hot and cold pack applications, nonsteroidal anti-inflammatory drugs, administration of calcium and vitamin D were initiated. After the treatment, all patients recovered fully.

Post-operative superficial wound infection and delayed healing were present in four patients. They were treated with local wound care, debridement, changing dressing daily with povidone iodine and administration of intravenous antibiotics (first generation cephalosporin) for two weeks according to the bacterial culture results. One patient developed skin flap necrosis at the corner of the lateral L-shaped incision. Debridement of the necrotic tissue yielded a good result.

Thirteen patients (39.4\%) recovered normally; eleven patients (33.4\%) recovered with subtalar arthrosis; nine (27.3\%) patients recovered with mild subtalar arthrosis.

There were no significant differences between those who had accompanies injuries and those who did not in terms of age $(p=0.345)$, sex $(p=0.539)$, duration of follow-up $(p=0.646)$, complication rate $(p=0.797)$ and final outcome distribution $(p=0.309)$.

The median AOFAS hindfoot score was $84.4 \pm 9.0$ points (range: 68-97) at the last follow-up. According to this score, 13 patients (39.4\%) had excellent scores, 11 patients (33.3\%) had good results and nine patients (27.3\%) had fair results.

\section{Table 1. The mechanism of trauma}

Motor vehicle injury

$1(3 \%)$

Fall from height $28(84.8)$

Work accident $2(6.1 \%)$

Suicide (fall from height) $2(6.1 \%)$ 
The AOFAS scores were significantly different between patients who had accompanying injuries and those who did not. In patients who did not have accompanying injuries, AOFAS scores were significantly higher $(p=0.036)$. The demographic, functional and clinical detailed evaluations of the two groups are summarised in Table 2.

The mean preoperative and post-operative Böhler angles were

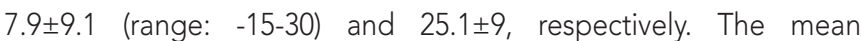
preoperative and post-operative Gissane angles were $143.1 \pm 13.1$ (range: 110-170) and 120 11.2 (range: 104-145), respectively.

The preoperative Böhler angle was not significantly different between the groups $(p=0.772)$. In patients with no accompanying injuries, the post-operative Böhler angle was significantly higher than in those with accompanying injuries $(p=0.007)$. In both groups, the post-operative Böhler angle increased significantly compared with the preoperative outcomes $(p<0.000)$. The preoperative $(p=0.630)$ and postoperative ( $p=0.241)$ Gissane angles did not differ significantly between the groups. The post-operative Gissane angle decreased significantly compared with the preoperative score in both groups $(p<0.000)$. The radiological results of the patients are summarised in Table 3.

Table 2. Demographic features, functional scores and clinical results of the patients according to accompanying injury

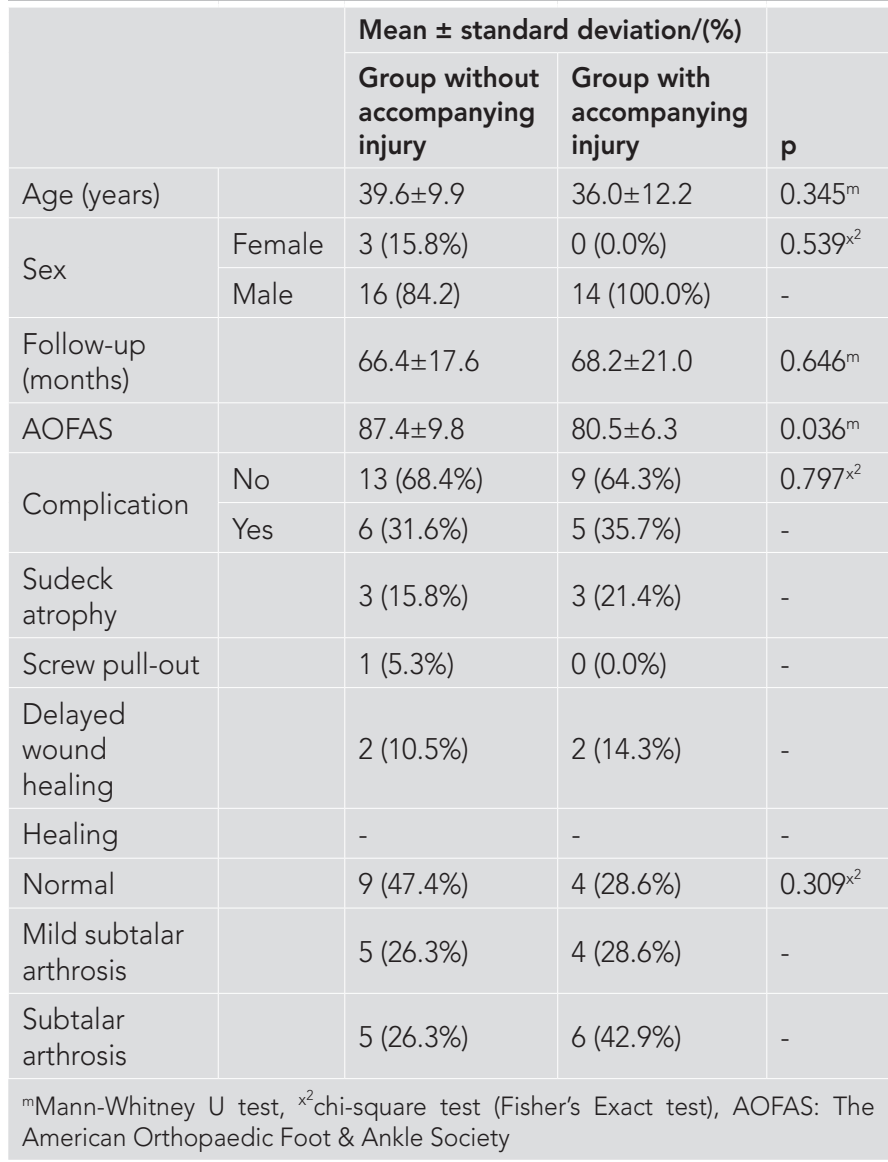

\section{DISCUSSION}

Displaced intra-articular calcaneus fractures are common injuries. However, their surgical treatment remains challenging. Anatomical reduction is the crucial approach for successful management of calcaneal fractures (4). Surgical treatment with the anatomical reduction of the calcaneal facets and restoration of overall calcaneal anatomy offer better clinical and functional results (7). The main goal of managing these fractures is to restore the congruity of the posterior calcaneal facet. Calcaneus height and width, varus-valgus alignment and calcaneocuboid involvement should be evaluated at the same time. Precise reduction of the subtalar joint may permit earlier mobilisation of the ankle. Additionally, the restoration of the calcaneal structure preserves the Achilles tendon lever arm and midfoot biomechanics (4). In this study, stable fixation with complete anatomical reduction was preferred.

The extended lateral approach has been considered the standard approach for performing ORIF of displaced intra-articular calcaneus fractures (8). Long et al. (9) revealed that locking plate provides good stability for calcaneal fracture even in elderly patients with osteoporotic fractures, which allows weightbearing at an early stage without exerting any adverse effect on stabilisation. Despite the advantages of the standard technique, a less invasive technique, sinus tarsi approach, was popularised in the last decade (10). Schepers et al. (10) revealed that the sinus tarsi approach reduces the rate of wound complication and has a shorter operative time. However, this approach cannot provide joint height and axial alignment. Additionally, percutaneous arthroscopic calcaneal osteosynthesis seems to be a good option for displaced intra-articular calcaneal fractures as it has a low complication rate. Long-term randomised controlled studies are needed to prove the effectiveness of this technique (11). We prefer lateral extensile approach and locking compressive plate to ensure anatomical calcaneus reduction and rigid fixation. Therefore, we provided early mobilisation, and we did not have

Table 3. Radiological evaluation of the patients with and without accompanying injury

\begin{tabular}{|c|c|c|c|}
\hline & $\begin{array}{l}\text { Group without } \\
\text { accompanying } \\
\text { injury }\end{array}$ & $\begin{array}{l}\text { Group with } \\
\text { accompanying } \\
\text { injury }\end{array}$ & $\begin{array}{l}\text { Group } \\
\text { difference } \\
p \text { value }\end{array}$ \\
\hline \multicolumn{4}{|c|}{ Böhler angle (point) } \\
\hline Preoperative & $8.0 \pm 10.2$ & $7.5 \pm 6.1$ & $0.772^{\mathrm{m}}$ \\
\hline Post-operative & $26.9 \pm 9.4$ & $21.0 \pm 7.1$ & $0.007^{m}$ \\
\hline $\begin{array}{l}\text { Preoperative/ } \\
\text { post-operative } \\
\text { difference p value }\end{array}$ & $0.000^{w}$ & $0.000^{w}$ & - \\
\hline \multicolumn{4}{|c|}{ Gissane angle (point) } \\
\hline Preoperative & $141.6 \pm 14.5$ & $146.5 \pm 8.9$ & $0.63^{m}$ \\
\hline Post-operative & $118.6 \pm 10.8$ & $123.3 \pm 12.0$ & $0.241^{m}$ \\
\hline $\begin{array}{l}\text { Preoperative/ } \\
\text { post-operative } \\
\text { difference p value }\end{array}$ & $0.000^{w}$ & $0.000^{w}$ & - \\
\hline
\end{tabular}


any wound healing problems, which could be a serious problem in the mid-term follow-up.

Intra-articular calcaneus fractures are severe, high-energy injuries with the potential for considerable morbidity, given the high rate of concomitant orthopaedic injuries. These highenergy fractures are accompanied by a significant disability and notable socioeconomic burden (12). In accordance with other series (13), most of our patients were males in their thirties with a history of falling from a height as the most common type of injury. Simultaneously, the presence of injuries accompanied by these high-energy injuries and bilateral fractures are among the important factors affecting the treatment and follow-up period. Agren et al. (14) stated that the patient's demographics should be considered when choosing the treatment option. Patients with disadvantageous prognostic factors should be presented, and realistic expectations were revealed. These fractures mostly affect young active male patients; for this reason, the socioeconomic importance should not be overlooked. The patient should return to the preinjury condition as soon as possible.

Mitchell et al. (15) discussed the mechanism of injury and associated injuries of calcaneus fractures. They found that more than $70 \%$ of fractures had resulted from falling from a height. Myerson and Quill (16) also discussed the injury mechanism, and they revealed that fractures resulted from a fall from a height in 33 (77\%) patients and a motor vehicle accident in $6(14 \%)$ patients. Similarly, in our study, falling from a height is the predominant cause. We think that open fractures are more common in motor vehicle injuries. Since we included closed fractures in this study, we think that falling from a height may be the reason why it is frequently included in the aetiology. We presented accompanying injuries in 14 patients (42.4\%). In our study, more than $80 \%$ of the aetiology was falling from a height. We think that accompanying injuries are frequently encountered due to the aetiology of such a high-energy injury.

Böhler angle is the most important prognostic radiological parameter, which shows the compression and deformity after trauma. Restoration of Böhler angle is associated with better outcomes (17). Researchers have revealed that the restoration of Böhler angle and quality of the subtalar joint reduction predict the outcome for treating displaced intra-articular calcaneal fractures, regardless of the treatment modality $(18,19)$. Gotha and Zide (7) revealed that restoration of Böhler angle at the operation, regardless of the angle at presentation, may be the better predictor of results over the time. When the radiological parameters were evaluated, in patients with isolated fractures, Böhler angle was higher than in patients with bilateral fractures and those with additional in the last follow-up in our study. This was thought to be due to axial loading during mobilisation. We recommend avoiding early axial loading and bone graft support in patients with bilateral fractures and those who have accompanying injuries.

In this study, of the 33 patients, 24 achieved good-to-excellent results according to AOFAS scores. The finding was comparable to the results of previous studies (20). In the group with no accompanying injuries, the AOFAS score was higher than in the group with accompanying injuries. This result is related to the rehabilitation process in the post-operative period. We concluded that, in the presence of accompanying injuries, the rehabilitation process is prolonged and this leads to negative effects on the functional outcomes.

Yu et al. (21) revealed that the timing of the surgery, preference of the surgical exposure, reduction of the fracture and perioperative management should be planned carefully, so that the complication rates could be reduced, which is in agreement with our results. The patients were operated on within five days on average, which is consistent with other series (13). In our study, complications were observed in 11 patients (33.3\%), including Sudeck's atrophy, superficial wound infection and delayed healing and screw cutout. Researchers revealed that wound healing problems occurred in $25-32 \%$ of the patients in whom lateral exposure was preferred $(22,23)$. Therefore, a higher incidence of wound problems arises due to this technique. These wound problems do not affect the mild-term functional clinical outcomes.

Bone grafting is a common procedure in calcaneus fracture restoration (24). Bone graft or bone graft substitute can be placed in the defects to provide additional stability for elevating the articular segment and prevent late collapse. The choice of graft material can include autogenous bone graft, allograft cancellous chips, bone graft substitutes (Pro-Osteon, DBX bone matrix, Norian SRS) or injectable calcium phosphate cement (25). Bone graft substitutes have been popularised to prevent complications associated with autogenous bone harvest. Bone grafts provide structural support for calcaneus anatomic restoration and may allow earlier weight-bearing. Malreduction could be prevented by incorporating into the bone substitutive of the posterior calcaneal facet. We used bone graft substitutes in all of the bilateral calcaneus fractures. We also used bone graft substitutes in unilateral cases when it was necessary. Schildhauer et al. (26) treated 32 patients with ORIF supplemented by calcium phosphate cement. Patients showed no loss of reduction. In accordance with the literature, we did not experience a loss of reduction in our patients. Patients with bilateral calcaneus fractures could be supported with a graft to sustain anatomical reduction and additional stability.

\section{Study Limitations}

The study has a limited number of patients. Radiological parameters in early post-operative and final follow-up were not compared. The relatively short follow-up period and unavailability of quality-of-life assessment constitute the weak points of the study.

\section{CONCLUSION}

Accompanying injuries in the calcaneus will adversely affect the radiological results and functional outcomes. During the management of calcaneus fractures, the accompanying injuries and their negative effects on the healing process should be taken into consideration. 
Ethics Committee Approval: This study was approved by the Clinical Research Ethics Committee of İstanbul Training and Research Hospital (decision no: 886, date: 25.11.2016).

Informed Consent: Informed consent was obtained from the patients.

Peer-review: Externally peer-reviewed.

Author Contributions: Surgical and Medical Practices - E.Ç., A.B.; Concept - E.Ç., A.B.; Design - E.Ç., A.B.; Data Collection and/or Processing - E.Ç.; Analysis and/or Interpretation - E.Ç.; Literature Search - E.Ç., A.B.; Writing - E.Ç., A.B.

Conflict of Interest: The authors have no conflict of interest to declare.

Financial Disclosure: The authors declared that this study has received no financial support.

\section{REFERENCES}

1. Cohen M: Calcaneal fractures. Foot Ankle Surg 2001; 2: 1819-63.

2. Dhillon MS, Bali K, Prabhakar S. Controversies in calcaneus fracture management: a systematic review of the literature. Musculoskelet Surg 2011; 95: 171-81.

3. Sanders R, Fortin P, DiPasquale T, Walling A. Operative treatment in 120 displaced intraarticular calcaneal fractures. Results using a prognostic computed tomography scan classification. Clin Orthop Relat Res 1993; 87-95.

4. Asik M, Sen C, Bilen FE, Hamzaoglu A. [Surgical management of intraarticular calcaneus fractures]. Acta Orthop Traumatol Turc 2002; 36: 35-41. (Turkish)

5. Sharr PJ, Mangupli MM, Winson IG, Buckley RE. Current management options for displaced intra-articular calcaneal fractures: non-operative, ORIF, minimally invasive reduction and fixation or primary ORIF and subtalar arthrodesis. A contemporary review. Foot Ankle Surg 2016; 22: $1-8$.

6. Kitaoka HB, Alexander IJ, Adelaar RS, Nunley JA, Myerson MS, Sanders M. Clinical rating systems for the ankle-hindfoot, midfoot, hallux, and lesser toes. Foot Ankle Int 1994; 15: 349-53.

7. Gotha HE, Zide JR. Current controversies in management of calcaneus fractures. Orthop Clin North Am 2017; 48: 91-103.

8. Gougoulias N, Khanna A, McBride DJ, Maffulli N. Management of calcaneal fractures: systematic review of randomized trials. Br Med Bull 2009; 92: 153-67.

9. Long C, Fang Y, Huang FG, Zhang H, Wang GL, Yang TF, et al. Sanders II-III calcaneal fractures fixed with locking plate in elderly patients. Chin J Traumatol 2016; 19: 164-7.

10. Schepers T, Backes M, Dingemans SA, de Jong VM, Luitse JSK. Similar anatomical reduction and lower complication rates with the sinus tarsi approach compared with the extended lateral approach in displaced intra-articular calcaneal fractures. J Orthop trauma 2017. 31: 293-8.
11. Pastides PS, Milnes L, Rosenfeld PF. Percutaneous arthroscopic calcaneal osteosynthesis: a minimally invasive technique for displaced intraarticular calcaneal fractures. J Foot Ankle Surg 2015; 54: 798-804.

12. Coughlin MJ. Calcaneal fractures in the industrial patient. Foot Ankle Int 2000; 21: 896-905

13. Jain S, Jain AK, Kumar I. Outcome of open reduction and internal fixation of intraarticular calcaneal fracture fixed with locking calcaneal plate. Chin J Traumatol 2013; 16: 355-60.

14. Agren PH, Mukka S, Tullberg T, Wretenberg P, Sayed-Noor AS. Factors affecting long-term treatment results of displaced intraarticular calcaneal fractures: a post hoc analysis of a prospective, randomized, controlled multicenter trial. J Orthop Trauma 2014; 28: 564-8.

15. Mitchell MJ, McKinley JC, Robinson CM. The epidemiology of calcaneal fractures. Foot (Edinb) 2009; 19: 197-200.

16. Myerson M, Quill GE. Late complications of fractures of the calcaneus. J Bone Joint Surg Am 1993; 75: 331-41.

17. Basile A. Operative versus nonoperative treatment of displaced intraarticular calcaneal fractures in elderly patients. J Foot Ankle Surg 2010; 49: 25-32.

18. Buckley R, Tough S, McCormack R, Pate G, Leighton R, Petrie D, et al. Operative compared with nonoperative treatment of displaced intraarticular calcaneal fractures: a prospective, randomized, controlled multicenter trial. J Bone Joint Surg Am 2002; 84: 1733-44.

19. Loucks C, Buckley R. Bohler's angle: correlation with outcome in displaced intra-articular calcaneal fractures. J Orthop Trauma 1999; 13: 554-8.

20. Cao L, Weng W, Song S, Mao N, Li H, Cai Y, et al. Surgical treatment of calcaneal fractures of Sanders type II and III by a minimally invasive technique using a locking plate. J Foot Ankle Surg 2015; 54: 76-81.

21. Yu X, Pang QJ, Chen L, Yang CC, Chen XJ. Postoperative complications after closed calcaneus fracture treated by open reduction and internal fixation: a review. J Int Med Res 2014; 42: 17-25.

22. Folk JW, Starr AJ, Early JS. Early wound complications of operative treatment of calcaneus fractures: analysis of 190 fractures. J Orthop Trauma 1999; 13: 369-72

23. De Groot R, Frima AJ, Schepers T, Roerdink WH. Complications following the extended lateral approach for calcaneal fractures do not influence mid- to long-term outcome. Injury 2013; 44: 1596-600.

24. Fitzgibbons TC, Hawks MA, McMullen ST, Inda DJ. Bone grafting in surgery about the foot and ankle: indications and techniques. J Am Acad Orthop Surg 2011; 19: 112-20

25. Younger EM, Chapman MW. Morbidity at bone graft donor sites. J Orthop Trauma 1989; 3: 192-5.

26. Schildhauer TA, Bauer TW, Josten C, Muhr G. Open reduction and augmentation of internal fixation with an injectable skeletal cement for the treatment of complex calcaneal fractures. J Orthop Trauma 2000; 14: 309-17. 